\title{
Análise da temperatura superficial de uma lagoa costeira integrada com dados orbitais e micrometeorológicos na passagem de sistemas frontais
}

\author{
Surface temperature analysis of a coastal lagoon integrated with orbital and \\ micrometeorological data during the passage of front systems \\ Marilei Bender Xavier¹, Sílvia Beatriz Alves Rolim², Gervásio Annes Degrazia³, \\ Waterloo Pereira Filho ${ }^{4}$ \\ ${ }^{1}$ Doutora, Docente da Universidade de Santa Cruz, RS, Brasil \\ ${ }^{2}$ Doutora , Docente da Universidade Federal do Rio Grande do Sul, RS, Brasil \\ ${ }^{3,4}$ Pós-Doutor, Docente da Universidade Federal de Santa Maria, RS, Brasil
}

\begin{abstract}
Resumo
Corpos d'água possuem uma dinâmica própria com diferentes características que sofrem variações com a passagem dos sistemas frontais. Estes sistemas aquáticos são influenciados pela temperatura do ar, vento e outras grandezas meteorológicas. O presente estudo apresenta um método para medir a temperatura superficial de uma lagoa costeira situada no extremo sul do Brasil utilizando imagens orbitais. Durante os meses de inverno de 2006 (junho, julho e agosto) analisou-se a temperatura de superfície da lagoa na ocorrência de seis sistemas frontais frios que passaram no Estado do Rio Grande do Sul. A temperatura de superfície foi estimada a partir do produto MOD11Temperatura de Superfície e Emissividade, do sensor Moderate Resolution Imaging Spectroradiometer (MODIS), a bordo da plataforma orbital EOS-TERRA. Os resultados evidenciaram que a temperatura da lagoa diminui após a passagem de frentes frias e é influenciada pelo vento e pela temperatura ambiente. As temperaturas máximas registradas na região norte da lagoa são, em média, $5^{\circ} \mathrm{C}$ mais altas na entrada da frente do que na saída das frentes frias. Este aumento é relacionado à entrada de nutrientes oriundos do banhado da reserva do Taim. A presente análise mostra que as medidas de temperatura superficial em lagoas, obtidas pelo sensor MODIS, constitui uma ferramenta viável e eficiente para estabelecer relações com o campo de vento originado durante a passagem de sistemas frontais.
\end{abstract}

Palavras-chave: Sensoriamento remoto. Sistemas frontais. Micrometeorologia

\begin{abstract}
Water bodies have a very dynamic with different characteristics that undergo changes with the passage of frontal systems. These water systems are influenced by the air temperature, wind and other meteorological quantities. This study presents a method to measure the surface temperature of a coastal lagoon located in southern Brazil using satellite images. During the winter months of 2006 (June, July and August) the surface temperature of the pond was analyzed in the occurrence of six cold frontal systems that passed in the state of Rio Grande do Sul. Surface temperature was estimated from the product MOD11-Surface Temperature and Emissivity, of the sensor Moderate Resolution Imaging Spectroradiometer (MODIS) aboard the EOS-TERRA orbital platform. The results showed that the temperature of the pond decreases after the passage of cold fronts and can be influenced by wind and ambient temperature. The maximum temperatures recorded in the northern region of the lagoon are on average $5{ }^{\circ} \mathrm{C}$ higher at the front entrance than at the exit of cold fronts. This increase is related to the input of nutrients coming from the marsh of Taim. This analysis shows that measures of surface temperature in ponds, obtained by the MODIS sensor, are a feasible and efficient tool to establish relations with the wind originated during the passage of frontal systems.
\end{abstract}

Keywords: Remote Sensing. Frontal Systems. Micrometeorology 


\section{Introdução}

A relação entre parâmetros micrometeorológicos e a variação da temperatura superficial de ecossistemas aquáticos permite compreender alguns problemas ambientais em lagoas costeiras. Neste aspecto, pode-se prever o efeito de impactos ambientais em ecossistemas caracterizados por uma escala temporal geológica de curta duração. Os ecossistemas aquáticos que apresentam pequenas extensões, como lagoas que ocorrem na interface entre os oceanos e regiões continentais de terra firme (região costeira), são sensíveis às mudanças meteorológicas provocadas por sistemas frontais (Mohseni \& Stefan, 2001; Stefan et al., 2001; Mohseni et al., 2003). Tais mudanças meteorológicas ocorrem por influência da temperatura, velocidade do vento e de outras variáveis. Como consequência, os efeitos das mudanças climáticas sobre estes corpos d'água podem ser estudados quando se considera a variação sazonal de parâmetros em longos períodos de tempo. Portanto, é importante medir as temperaturas superficiais de lagoas e estabelecer as suas relações com os parâmetros micrometeorológicos (Justice et al., 2002; Dousset \& Gourmelon, 2003).

Apesar da importância de se realizar observações de parâmetros meteorológicos, a obtenção de medidas locais destas quantidades em corpos de água situados em terrenos acidentados é uma tarefa extremamente difícil. Por exemplo, coletar dados em meios aquáticos envolve uma logística complicada, uma vez que forçantes meteorológicos presentes no momento da coleta podem tornar inviável o procedimento de medida. Normalmente, as observações em lagoas são interrompidas por alterações súbitas na magnitude do vento. Uma técnica alternativa que permite medir as temperaturas superficiais de lagoas costeiras é baseada no emprego de dados orbitais (Ustin, 2004). Estes dados, obtidos a partir de sensores localizados em satélites, medem continuamente e de forma detalhada as temperaturas superficiais destes particulares ecossistemas aquáticos em diferentes situações meteorológicas. Tais padrões meteorológicos podem ser avaliados analisando-se as variações relacionadas aos fatores bióticos e as propriedades físico-químicas do ecossistema estudado (Tundisi et al., 2010). As observações das temperaturas superficiais, mediadas em uma escala de tempo, podem revelar padrões importantes de mudanças climáticas. Além disso, várias comunidades biológicas, como algumas espécies de peixes e algas são sensíveis a variações da temperatura de lagoas (Rodrigues, 2009). Como consequencia, a medida de temperatura superficial extraída a partir de imagens orbitais é uma das principais variáveis para o entendimento de fenômenos relacionados ao estudo ambiental de lagoas. Um parâmetro micrometeorológico que influencia as flutuações da temperatura superficial de lagoas é o vento. Com a passagem de um sistema frontal pode ocorrer diminuição ou aumento tanto da radiação solar incidente quanto da temperatura do ar e da velocidade do vento. Na região sul do Brasil, no Estado do Rio Grande do Sul (RS) estão presentes particulares lagoas caracterizadas por tempos geológicos pequenos. Tais sistemas aquáticos, extremamente dependentes de mudanças ambientais, estão localizados na interface entre o continente e o oceano.

O objetivo do presente estudo é empregar imagens orbitais para se medir de forma remota a temperatura superficial da lagoa da Mangueira, RS. Estas observações são realizadas durante a passagem de sistemas frontais no sul do Brasil. Um propósito complementar do estudo é analisar a magnitude e as direções dos ventos durante as passagens das frentes frias e relacionar estas quantidades com as flutuações da temperatura superficial da lagoa. Para a realização deste estudo foi empregada a metodologia do produto MOD11 do sensor MODIS e dados meteorológicos medidos na estação de Santa Vitória do Palmar, RS. O Produto MOD11 calcula a temperatura de superfície terrestre em função da radiância espectral e das medidas de emissividades nas bandas $31(10,78$ a 11,28 $\mu \mathrm{m})$ e $32(11,77$ a $12,27 \mu \mathrm{m})$ do infravermelho termal.

\section{Caracterização da Área de Estudo}

A lagoa Mangueira (Figura 1) é um ecossistema de água doce, constituído principalmente por um corpo de água raso, apresentando profundidades que variam entre 1,5 e $6 \mathrm{~m}$ (Delaney,1965). Ela possui uma forma alongada com $92 \mathrm{~km}$ de comprimento e com uma largura oscilando entre 2 e $10 \mathrm{~km}$. A sua área aproximada é de $820 \mathrm{~km}^{2} \mathrm{e}$ se estende na zona costeira do sul do Brasil, sendo localizada entre os municípios de Santa Vitória do Palmar e Rio Grande. Esta lagoa constitui uma parte do Sistema Hidrológico da Reserva do Taim, abrangendo uma área total de $2254 \mathrm{Km}^{2}$. Ela é rodeada por uma variedade de habitats, tais como praias, florestas, pastagens e dunas de zonas úmidas. Atualmente, a orizicultura constitui a principal atividade econômica da região e a extração de água da lagoa Mangueira para as lavouras tem alterado

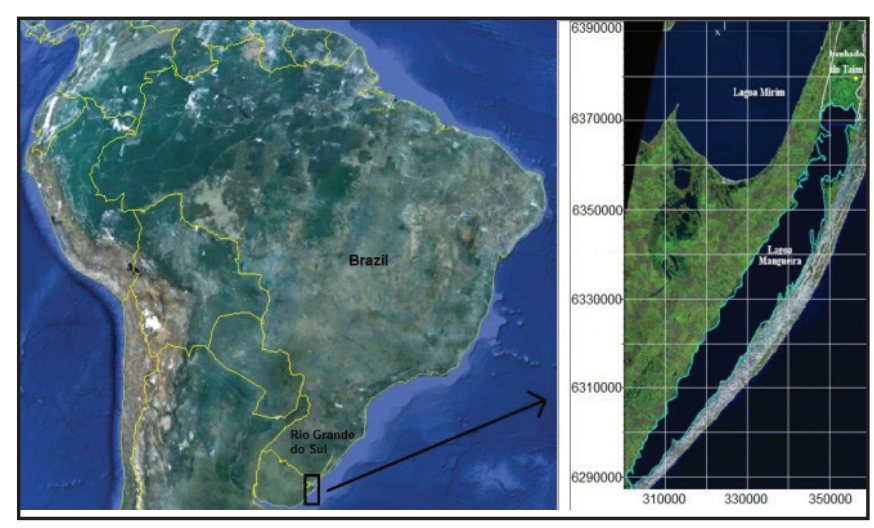

Figura 1. Área de estudo, Lagoa Mangueira, RS, Brasil 
o seu regime hidrológico. Ambientes lacustres, como o sistema formado pela lagoa Mangueira são transitórios, pois dependem da dinâmica geológica, fluvial e do clima da região. Neste sentido, a complexidade e o balanço ecológico delicado dos ambientes que formam a paisagem do entorno desta lagoa são sensíveis à ação antrópica fomentada pela exploração agrícola (orizicultura).

\subsection{Aspectos da Vegetação e Vento na Lagoa Mangueira}

Na região costeira do RS, a vegetação e o vento são dois agentes responsáveis pelas alterações na hidrodinâmica dos sistemas lacustres. Segundo Rodrigues (2009), a lagoa Mangueira possui diferentes características bióticas e apresenta distintas propriedades físico-químicas da água. Tais mudanças são demonstradas por gradientes espaciais e temporais das diferentes propriedades. Os estudos de Rodrigues (2009) mostram que na região norte há um aumento na concentração de sólidos totais que está associada à entrada de nutrientes e matéria orgânica originadas do banhado que constitui a reserva do Taim. Considerando-se os ventos, Fragoso Jr. et al. (2008) mostram que os escoamentos de ar predominantes na lagoa Mangueira são de nordeste para sudoeste (velocidade média de $8 \mathrm{~m} / \mathrm{s}$ ), seguindo o eixo principal da lagoa. As passagens de frentes frias são mais comuns no inverno do que no verão. Como consequência, o regime de ventos dominantes move as águas superficiais em direção ao sul da lagoa Mangueira (DELANEY, 1965; GODOLPHIN, 1976; ZELTZER, 1976; TOMAZELLI, 1993). Este campo de vento gera uma energia suficiente para que ocorra uma ressuspensão de sedimentos finos, resultando um longo fetch que pode alcançar $90 \mathrm{~km}$.

\section{Metodologia e Medidas de Temperatura de Superfície Através de Dados Orbitais}

Um grande número de trabalhos científicos analisa e discute a variação da temperatura superficial de diferentes alvos por meio de dados de emissividade e sensores remotos a bordo de plataformas orbitais. Dentre eles destacam-se os trabalhos de Becker \& Li (1990), Kerr et al. (1992), Prata (1994), Ulivieri et al. (1994), Sobrino, Li \& Becker (1996), Dash et al. (2002). Vários sensores a bordo de satélites possuem dados na região do infravermelho termal, como o sensor Moderate Resolution Imaging Spectroradiometer (MODIS) do satélite EOS-TERRA. Dentre os vários produtos que podem ser obtidos do sensor MODIS pode-se destacar o produto MOD11- (Temperatura de Superfície Terrestre e Emissividade). Uma das importantes aplicações do produto MOD11 é a validação e o melhoramento de modelos de previsão meteorológicos (Strahler et al., 1999). Os dados orbitais utilizados neste estudo, referentes à temperatura superficial da lagoa Mangueira, foram obtidos do produto MOD11
(Temperatura de Superfície e Emissividade) do sensor MODIS. O satélite TERRA foi lançado em dezembro de 1999, coletando dados a partir de fevereiro de 2000, com o horário de passagem as 10:30h sobre América do Sul. As imagens foram adquiridas através do endereço eletrônico http://reverb.echo.nasa.gov/reverb nas datas das passagens das frentes frias dos meses de inverno (junho, julho e agosto) de 2006.

A base fundamental do sensoriamento remoto para estimar a temperatura de superfície da terra emprega a função de Planck. A taxa emitida em cada comprimento de onda, chamada de emitância ou poder emissivo espectral $\left[\mathrm{W} / \mathrm{m}^{2} \mu \mathrm{m}\right]$ do corpo negro é dado por:

$$
M(\lambda, T)=\frac{C_{1}}{\lambda^{5}\left[\exp \left(C_{2} / \lambda T\right)-1\right]}
$$

onde $M$ é a emitância espectral; $C_{1}=3,74 \times 10^{-8}\left(\mathrm{Wm}^{2}\right)$, $\mathrm{C}_{2}=1,44 \times 10^{4}(\mu \mathrm{mK})$ são constantes, $\mathrm{T}$ é a temperatura (K) e $\lambda$ é o comprimento de onda medido $(\mu \mathrm{m})$.

Integrando a equação (1) em todos os comprimentos de onda $(\lambda)$ temos a emitância de um corpo negro que é representado por:

$$
M=\sigma T^{4}
$$

onde $\sigma=5,669 \times 10^{-8} \mathrm{Wm}^{-2} \mathrm{~K}^{-4}$ (constante de Stefan-Boltzmann).

A equação (2) representa a radiação de um corpo ideal ou corpo negro, na qual é baseada nos princípios da termidinâmica, se e somente se, a superfície for um emissor perfeito.

Um corpo negro a uma determinada temperatura emite radiação por unidade de comprimento, em todos os comprimentos de onda próximos de zero ao infinito, mas não a mesma quantidade de radiação em cada comprimento de onda. Assim, a razão entre as emitâncias reais e de corpo negro é o que se denomina de emissividade do corpo real e é expressa pela seguinte razão:

$$
\varepsilon_{\lambda}=\frac{E_{\lambda}}{M_{\lambda}}
$$

onde $E_{\lambda}$ é a emitância em um comprimento de onda $(\lambda)$ de uma superfície real a uma temperatura $\mathrm{T}$ e $M_{\lambda}$ é a emitância espectral (emissor perfeito).

As observações de temperatura da superfície terrestre fornecidas pelo produto MOD11 do sensor MODIS (na resolução de $1 \mathrm{~km}$ ) são calculadas a partir do algoritmo "generalized split-window" (Wan \& Dozier, 1996), o qual assume um conhecimento a priori da emissividade. As medidas de emissividades das bandas 31(10,78 a 11,28 $\mu \mathrm{m})$ e 32(11,77 a 12,27 $\mu \mathrm{m})$ são obtidas com base em uma classificação, na qual o pixel da imagem é identificado com 14 classes de uso da terra pré-definidas e com os 
valores correspondentes de emissividade (SNYDER et al., 1998). A partir do conhecimento a priori da emissividade, o algoritmo "generalized split-window" realiza a correção atmosférica baseado na absorção diferencial entre as duas bandas termais (31 e 32) do MODIS (LIANG, 2004). Segundo Wan \& Dozier (1996) a estimativa da temperatura de superfície através do algoritmo generalized split-window do sensor MODIS é dada por:

$$
T_{S}=\left(A_{1}+A_{2} \frac{1-\varepsilon}{\varepsilon}+A_{3} \frac{\Delta \varepsilon}{\varepsilon^{2}}\right) \frac{T_{3}+T_{3}}{2}+\left(B_{1}+B_{2} \frac{1-\varepsilon}{\varepsilon}+B_{3} \frac{\Delta \varepsilon}{\varepsilon^{2}}\right)\left(T_{3}-T_{3}\right)+C
$$

na qual, a temperatura da supefície terrestre, $\mathrm{T}_{31}$ e $\mathrm{T}_{32}$ são as temperaturas de brilho nas banda 31 e 32 do MODIS; $\varepsilon=$ $0,5\left(\varepsilon_{31}+\varepsilon_{32}\right)$ e $\Delta \varepsilon=\varepsilon_{31}-\varepsilon_{32}$ são as emissividades da superfície nas bandas 31 e 32 do MODIS; C, A1, A2, A3, B1, B2, B3 são os coeficientes de regressão. Esta estimativa é uma equação de regressão que utiliza a temperatura de brilho dos canais do infravermelho termal para calcular a temperatura de superfície. Os coeficientes de regressão são derivados usando um modelo de transferência radiativa com características atmosféricas obtidas a partir de dados originados de 12 perfis globais de temperatura atmosférica (WAN; DOZIER, 1996).

\subsection{Medidas de Temperatura de Superfície e Dados Micrometeorológicos na Lagoa Mangueira}

Os dados originais do pixel de temperatura de superfície do produto MOD11 fornecidos em Kelvin foram convertidos para graus Celsius através do cálculo: $\mathrm{T}^{\circ} \mathrm{C}$ $=\left(P_{i x}^{*} 0,02\right)-273$, no qual, $\mathrm{T}^{\circ} \mathrm{C}$ é o valor do pixel em graus Celsius, Pix é o valor original do pixel em graus Kelvin e 0,02 é a constante do fator de multiplicação para imagem diurna do produto MOD11. Para a observação da variação de temperatura na lagoa Mangueira foi traçado um perfil longitudinal (PL) nas imagens de temperatura do sensor MODIS e uma máscara da área total da superfície da lagoa (Figura 2). O perfil longitudinal foi traçado na direção Sul-Norte (SN) e a máscara

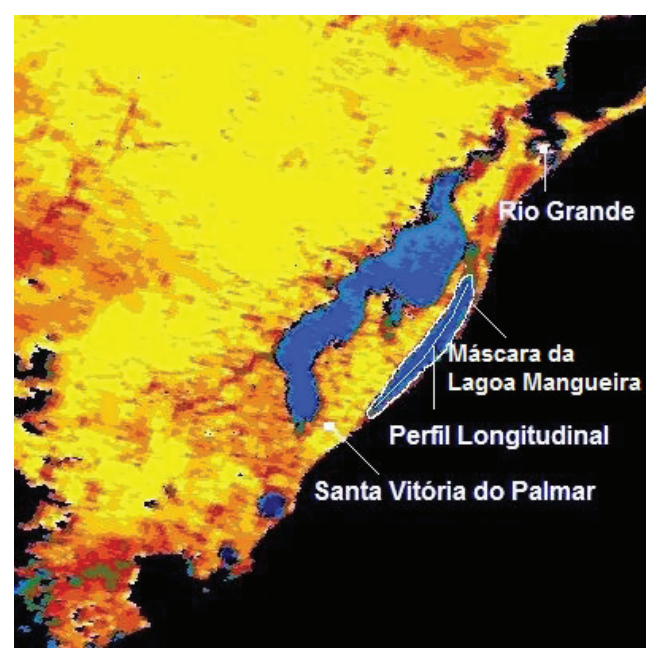

Figura 2 - Imagem do sensor MODIS e do PL de temperatura de superfície na Lagoa Mangueira foi traçada a partir dos limites que separam a água da lagoa e a terra firme. O posicionamento do perfil PL na imagem da Figura 2 é mostrado na Tabela 1.

Tabela 1 - Posicionamento dos pontos do perfil PL de temperatura de superfície traçado na lagoa Mangueira

\begin{tabular}{|c|c|c|c|c|}
\hline \multirow{3}{*}{ PL } & \multicolumn{2}{|c|}{ Ponto Inicial } & \multicolumn{2}{c|}{ Posição Final } \\
\cline { 2 - 5 } & Latitude & Longitude & Latitude & Longitude \\
\cline { 2 - 5 } & $33^{\circ} 30^{\prime} 40.2^{\prime \prime} \mathrm{S}$ & $53^{\circ} 6^{\prime} 41.2^{\prime \prime} \mathrm{W}$ & $32^{\circ} 35^{\prime} 10.5^{\prime \prime} \mathrm{S}$ & $52^{\circ} 31^{\prime} 39.9^{\prime \prime} \mathrm{W}$ \\
\hline
\end{tabular}

Os dados meteorológicos da área de estudo foram extraídos da Estação Meteorológica de Observação de Superfície Convencional localizada em Santa Vitória do

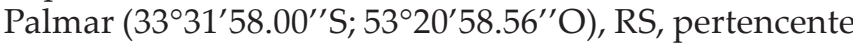
ao Instituto Nacional de Meteorologia (INMET), órgão do Ministério da Agricultura, Pecuária e Abastecimento, que coleta medidas diárias de variáveis climáticas. Neste estudo considera-se a velocidade do vento $(\mathrm{m} / \mathrm{s})$, a sua direção e a temperatura ambiente, $\mathrm{TA}\left({ }^{\circ} \mathrm{C}\right)$. Estas variáveis são coletadas em três horários distintos, $12 \mathrm{~h}, 18 \mathrm{~h}$ e $24 \mathrm{~h}$. Devido a homogeneidade e a topografia da região, as medidas na estação meteorológica são representativas do campo de vento da lagoa Mangueira. Durante os meses de junho, julho e agosto de 2006 foram selecionados 6 sistemas frontais frios que passaram na região sul do Brasil. Estes sistemas frontais foram selecionados do banco de dados do boletim do site do Instituto Nacional de Pesquisas Espaciais (INPE), (http://climanalise.cptec. inpe.br/ rclimanl/boletim/index0706.shtml\#).

Os meses de inverno no RS são caracterizados por dias encobertos na maior parte do período, com muita nebulosidade, o que dificultou a seleção das imagens das frentes frias. A Tabela 2 apresenta o resumo de duração de dias dos sistemas frontais, selecionados neste estudo, que chegaram na região sul do Brasil e que tiveram influência sobre a lagoa Mangueira. Neste resumo da Tabela 2 considerou-se o final da frente fria no dia em que se observou a imagem MODIS de céu limpo (após a passagem do sistema frontal por Santa Vitória do Palmar, RS.

As Tabela 2.1, 2.2 e 2.3, referentes aos meses de junho, julho e agosto, respectivamente, mostram a influência dos sistemas frontais desde suas chegadas em Santa Vitória do Palmar, RS (linha vermelha) até suas dissipações totais, a medida que avançam na trajetória (linhas pretas) para o norte do Brasil.

Tabela 2 - Período de duração dos Sistemas Frontais na área de estudo

\begin{tabular}{c|c|c|c}
\hline \multirow{2}{*}{ Mês /Ano } & $\begin{array}{c}\text { Sistema } \\
\text { Frontal } \\
\text { (SF) }\end{array}$ & $\begin{array}{c}\text { Dias de } \\
\text { duração do } \\
\text { SF }\end{array}$ & $\begin{array}{c}\text { Dia da } \\
\text { imagem } \\
\text { MODIS }\end{array}$ \\
\hline \multirow{2}{*}{ Junho/2006 } & $1^{\circ}$ & 9 a 11 & 8 e 12 \\
\cline { 2 - 4 } Julho/2006 & $2^{\circ}$ & 14 a 15 & 13 e 16 \\
\cline { 2 - 4 } & $3^{\circ}$ & 8 a 9 & 07 e 09 \\
\hline \multirow{2}{*}{ Agosto/2006 } & $4^{\circ}$ & 21 a 22 & 21 e 23 \\
\cline { 2 - 4 } & $5^{\circ}$ & 19 a 20 & 19 e 21 \\
\hline
\end{tabular}


Tabela 2.1 - Sistemas frontais do mês de junho de 2006

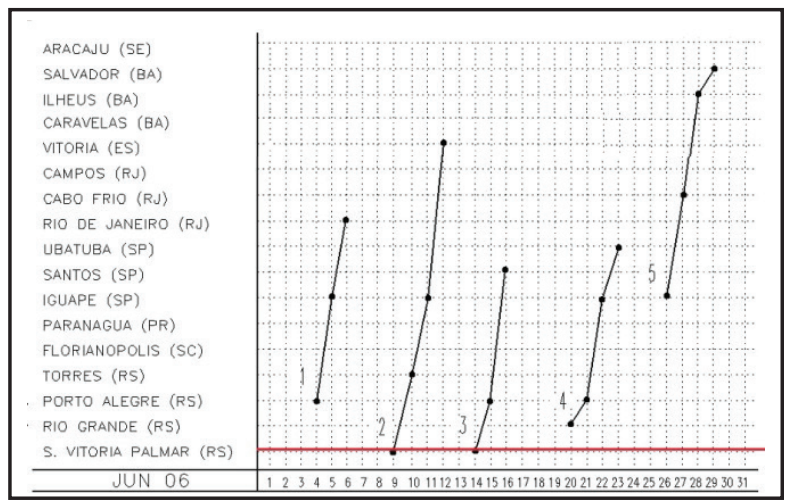

Fonte:http://climanalise.cptec.inpe.br/ rclimanl/boletim/edicoes/2006/jun/fig22.shtml.

Tabela 2.2 - Sistemas frontais do mês de julho de 2006

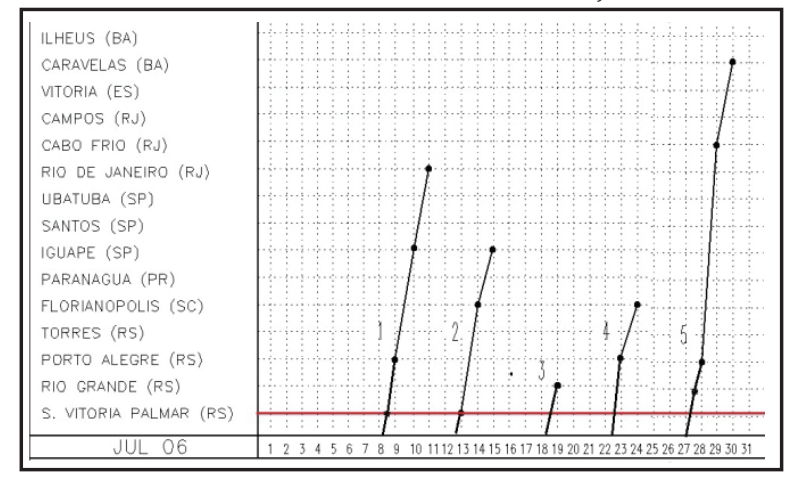

Fonte:http://climanalise.cptec.inpe.br/ rclimanl/boletim/edicoes/2006/jul/fig22.shtml

Tabela 2.3 - Sistemas frontais do mês de agosto de 2006

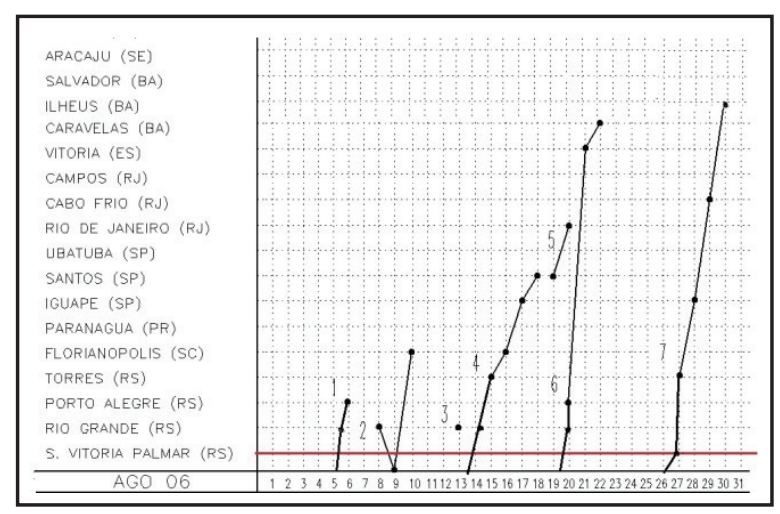

Fonte:http://climanalise.cptec.inpe.br/ rclimanl/boletim/edicoes/2006/ago/fig22.shtml

Baseado nas datas das passagens dos sistemas frontais das Tabelas, 2.1, 2.2 e 2.3, selecionaram-se dois sistemas frontais de cada mês viáveis de se obter as imagens com dados da temperatura de superfície. As imagens do sensor MODIS foram selecionadas, com base na cobertura de nuvens sobre a lagoa Mangueira. As imagens escolhidas representam dias de céu claro.
Selecionou-se uma imagem antes da chegada da frente fria e uma imagem após a passagem da frente fria. A Tabela 3 indica as frentes frias relacionadas com os dados micrometeorológicos, os dias em que as imagens foram selecionadas, a média da velocidade dos ventos e a média da temperatura ambiente $\mathrm{TA}\left({ }^{\circ} \mathrm{C}\right)$ para todo o período de duração da passagem dos sistemas frontais .

Tabela 3 - Datas das imagens MODIS correspondentes às frentes frias e associadas aos dados micrometeorológicos de Santa Vitória do Palmar, RS

\begin{tabular}{|c|c|c|c|c|c|c|c|}
\hline $\begin{array}{c}\text { Frente } \\
\text { Fria }\end{array}$ & Data & Hora & $\begin{array}{c}\text { TA } \\
\left({ }^{\circ} \mathrm{C}\right)\end{array}$ & $\begin{array}{l}\text { Vento } \\
(\mathrm{m} / \mathrm{s})\end{array}$ & $\begin{array}{c}\text { Direção } \\
\text { do } \\
\text { vento } \\
\end{array}$ & $\begin{array}{l}\text { Média } \\
\text { Vento } \\
(\mathrm{m} / \mathrm{s}) \\
\end{array}$ & $\begin{array}{c}\text { Média } \\
\text { TA } \\
\left({ }^{\circ} \mathrm{C}\right) \\
\end{array}$ \\
\hline \multirow{3}{*}{$\begin{array}{c}1^{\mathrm{a}} \\
\text { Entrada }\end{array}$} & \multirow{3}{*}{$08 / 06$} & 12 & 19,3 & 5 & NW & \multirow{6}{*}{4,1} & \multirow{6}{*}{12,0} \\
\hline & & 18 & 27,8 & 7 & NW & & \\
\hline & & 24 & 20,8 & 2 & NW & & \\
\hline \multirow{3}{*}{$\begin{array}{c}1^{\mathrm{a}} \\
\text { Saída }\end{array}$} & \multirow{3}{*}{$12 / 06$} & 12 & 6,7 & 0 & - & & \\
\hline & & 18 & 14,8 & 3 & $\mathrm{~N}$ & & \\
\hline & & 24 & 9,4 & 3 & $\mathrm{NE}$ & & \\
\hline \multirow{3}{*}{$\begin{array}{c}2^{\mathrm{a}} \\
\text { Entrada }\end{array}$} & \multirow{3}{*}{$13 / 06$} & 12 & 10,6 & 2 & NW & \multirow{6}{*}{1,7} & \multirow{6}{*}{12,0} \\
\hline & & 18 & 17,3 & 1 & NW & & \\
\hline & & 24 & 13,7 & 1 & NW & & \\
\hline \multirow{3}{*}{$\begin{array}{c}2^{\mathrm{a}} \\
\text { Saída }\end{array}$} & \multirow{3}{*}{$16 / 06$} & 12 & 6,8 & 2 & SW & & \\
\hline & & 18 & 12,0 & 2 & SW & & \\
\hline & & 24 & 5,6 & 1 & $\mathrm{~S}$ & & \\
\hline \multirow{3}{*}{$\begin{array}{c}3^{\mathrm{a}} \\
\text { Entrada }\end{array}$} & \multirow{3}{*}{$07 / 07$} & 12 & 13,6 & 2 & NW & \multirow{6}{*}{4,2} & \multirow{6}{*}{16,7} \\
\hline & & 18 & 24,6 & 3 & $\mathrm{NE}$ & & \\
\hline & & 24 & 18,0 & 6 & $\mathrm{NE}$ & & \\
\hline \multirow{3}{*}{$\begin{array}{c}3^{\mathrm{a}} \\
\text { Saída }\end{array}$} & \multirow{3}{*}{$09 / 07$} & 12 & 11,2 & 8 & $\mathrm{~W}$ & & \\
\hline & & 18 & 14,8 & 8 & $\mathrm{~W}$ & & \\
\hline & & 24 & 11,0 & 4 & $\mathrm{~W}$ & & \\
\hline \multirow{3}{*}{$\begin{array}{c}4^{\mathrm{a}} \\
\text { Entrada }\end{array}$} & \multirow{3}{*}{$21 / 07$} & 12 & 15,1 & 1 & $\mathrm{NE}$ & \multirow{6}{*}{2,4} & \multirow{6}{*}{16,4} \\
\hline & & 18 & 21,7 & 2 & SW & & \\
\hline & & 24 & 14,9 & 5 & SE & & \\
\hline \multirow{3}{*}{$\begin{array}{c}4^{a} \\
\text { Saída }\end{array}$} & \multirow{3}{*}{$23 / 07$} & 12 & 11,6 & 1 & SW & & \\
\hline & & 18 & 14,8 & 2 & $\mathrm{~S}$ & & \\
\hline & & 24 & 10,3 & 0 & - & & \\
\hline \multirow{3}{*}{$\begin{array}{c}5^{\mathbf{a}} \\
\text { Entrada }\end{array}$} & \multirow{3}{*}{$19 / 08$} & 12 & 9,4 & 2 & $\mathrm{~W}$ & \multirow{6}{*}{6,0} & \\
\hline & & 18 & 17,8 & 8 & NW & & \\
\hline & & 24 & 13,0 & 6 & NW & & 08 \\
\hline & & 12 & 6,6 & 6 & SW & & 9,8 \\
\hline Saída & $21 / 08$ & 18 & 12,2 & 4 & SW & & \\
\hline & & 24 & 6,5 & 1 & SW & & \\
\hline & & 12 & 16,0 & 1 & NW & & \\
\hline Entrada & $25 / 08$ & 18 & 25,6 & 0 & $\mathrm{C}$ & & \\
\hline & & 24 & 15,5 & 2 & $\mathrm{NE}$ & 4,0 & 136 \\
\hline & & 12 & 8,4 & 4 & SW & & 15,6 \\
\hline Saída & $28 / 08$ & 18 & 14,6 & 7 & SW & & \\
\hline & & 24 & 8,6 & 2 & SW & & \\
\hline
\end{tabular}

\section{Discussão e Análise dos Resultados}

As Figuras 3a, 3b; 4a, 4b; 5a, 5b; 6a, 6b; 7a, 7b; 8a, 8b representam as passagens iniciais e finais dos sistemas frontais que atingiram o sul do Brasil nos meses de junho, julho e agosto de 2006. Nesta figuras, a área da superfície da lagoa está evidenciada pelo traçado branco. As Figuras, 9, 10,11, 12, 13, 14, exibem o perfil PL de temperatura superficial da lagoa Mangueira (TSL). Estas figuras são confeccionadas a partir das imagens MODIS durante as passagens das frentes frias. Nestas figuras é representado, 
nas entradas e saídas das frentes, o comportamento da TSL ao longo da direção sul-norte. A Figura 9 representa a TSL na primeira frente fria. Pode-se observar que a TSL apresenta maiores flutuações na entrada da frente e aumenta subitamente na região norte da lagoa. Este aumento da TSL na parte norte também é observado nos demais perfis de entrada da frente fria (Figs 9, 11, 12 e 14). Esta temperatura mais elevada, observada ao norte, é caracterizada pela presença do parque da reserva natural Taim, que conecta com as águas da lagoa Mangueira. A passagem da segunda frente fria (Fig.10) exibe menores oscilações da TSL, particularmente, na entrada da frente fria. O perfil de saída desta frente mostra flutuações mais acentuadas na região sul da lagoa. Durante a passagem do terceiro sistema frontal (Fig. 11) são observadas flutuações maiores na entrada da frente, particularmente, na região central da lagoa, onde ocorre um decréscimo da TSL, em relação à parte sul e norte. Na saída desta frente a temperatura se estabiliza e aumenta no centro da lagoa, diferentemente das saídas das demais frentes frias. A quarta frente fria (Fig.12) apresenta TSL praticamente constante ao sul da lagoa e maiores flutuações ao centro e norte da lagoa. Na quinta frente fria, tanto na entrada como na saída da frente (Fig.13), a TSL apresenta pequenas flutuações na região sul e uma maior variação na região norte. Na sexta frente fria (Fig. 14) também é observado, na entrada e saída da frente, pequenas flutuações no sul e no centro e maiores flutuações no norte da lagoa.

As Tabelas 4 e 5 exibem, para as entradas e saídas dos sistemas frontais frios, diferentes valores e índices estatísticos da TSL do perfil PL e da área total (máscara) da lagoa, respectivamente. Estas quantidades são calculadas a partir dos valores de TSL observados pelo perfil traçado no eixo central da lagoa e dos valores gerais, medindo toda superfície da lagoa. Mais especificamente, nas entradas e saídas das frentes frias, esta tabela mostra os valores máximos e mínimos, os valores médios $(\overline{\$})$, os desvios padrões $\left(S_{T}\right)$ e os coeficientes de variações $(\mathrm{CV})$ da TSL. Os desvios padrões da temperatura superficial $S_{T}$ indicam a magnitude das flutuações desta variável em relação aos seus valores médios.

Através da análise estatística, observa-se que as maiores magnitudes das flutuações (os mais altos valores de $S_{T}$ ), ocorrem durante a passagem da terceira frente fria, seguida da primeira e da sexta frente fria. Estas flutuações apresentam uma variação da ordem de $3^{\circ} \mathrm{C}$ na TSL a partir do perfil PL(Tabela 4) e variação da ordem de $2^{\circ} \mathrm{C}$ considerando toda a superfície da lagoa (Tabela 5). Neste ponto é importante analisar a velocidade e a direção do vento juntamente com $\mathrm{TA}\left({ }^{\circ} \mathrm{C}\right)$ nos períodos de passagem das frentes.

A primeira entrada de frente fria (Fig. 9), inicia com uma magnitude de vento NW variando entre 5 e $7 \mathrm{~m} / \mathrm{s}$. Neste processo a TSL apresenta média de $17,35^{\circ} \mathrm{C}$ e CV de $17 \%$. Esta alta TSL pode ser associada com $\mathrm{TA}\left({ }^{\circ} \mathrm{C}\right)$ variando de 19 a $27^{\circ} \mathrm{C}$. Já a saída desta frente caracteriza- se por ventos mais fracos, da ordem de $3 \mathrm{~m} / \mathrm{s}$ e $\mathrm{TA}\left({ }^{\circ} \mathrm{C}\right)$ mais baixa, gerando queda da TSL e menor variação ao longo do perfil SN.

Durante a passagem da segunda frente fria (Fig.10), ocorre predominância de vento NW fraco com velocidade variando entre 1 a $2 \mathrm{~m} / \mathrm{s}$ e $\mathrm{TA}\left({ }^{\circ} \mathrm{C}\right)$ baixas. A saída desta frente é marcada pelo mudança da direção do vento $\mathrm{NW} / \mathrm{SW}$ ainda fraco com queda da $\mathrm{TA}\left({ }^{\circ} \mathrm{C}\right)$, gerando pequena queda da TSL.

A terceira frente fria (Fig. 11) mostra, particularmente, que a TSL aumenta após a passagem da frente fria na região central da lagoa. Comparando este resultado com os valores exibidos na Tabela 3, observa-se que a direção e a velocidade do vento, NW/NE, da ordem média de $4,2 \mathrm{~m} / \mathrm{s}$, durante este evento, provocaram as maiores flutuações nas medidas da TSL, com CV da ordem de $23,19 \%$. Ainda, analisando-se a TA $\left({ }^{\circ} \mathrm{C}\right)$ para este evento observam-se temperaturas altas, com média de $16,7^{\circ} \mathrm{C}$. Este fenômeno de aumento da TSL na saída da frente fria pode ser explicado pela alta temperatura ambiente constante ocorrida desde o início da chegada da frente. Neste caso, o tempo de relaxação de resposta da TSL não foi suficiente para que ocorresse o resfriamento da água, na saída desta frente fria.

A quarta frente fria (Fig. 12) apresenta pouca variação da TSL tanto na entrada como na saída da frente. Observa-se, pela Tabela 3, vento fraco caracterizado durante toda a passagem da frente como meandro direcional. $\mathrm{A} \mathrm{TA}^{\circ}(\mathrm{C})$ apresenta-se com pouca variação, com média de $16^{\circ} \mathrm{C}$. O CV de $13,07 \%$ justifica-se pelo aumento súbito da TSL ao norte da lagoa influenciada pelo banhado do Taim.

Na quinta frente fria (Fig. 13) a TSL é influenciada pelo forte vento NW e SW na entrada e saída da frente, respectivamente, média de $6 \mathrm{~m} / \mathrm{s}$ e baixas TA $\left({ }^{\circ} \mathrm{C}\right)$, média de $9,8^{\circ} \mathrm{C}$ durante toda a passagem do sistema frontal. A TSL sofreu queda na saída desta frente da ordem média de $2^{\circ} \mathrm{C}$ e gerou maior variação, $\mathrm{CV}$ de $6,42 \%$.

Durante a passagem da sexta frente fria (Fig.14), ocorre predominância de vento $\mathrm{SW}$ com velocidade variando entre 2 a $6 \mathrm{~m} / \mathrm{s}$, ocorrendo a terceira maior variação da TSL, CV de 15,9\% na entrada da frente. A saída desta frente é marcada pelo vento SW com magnitude máxima de $7 \mathrm{~m} / \mathrm{s}$ gerando queda da TSL ao longo de todo percurso SN da lagoa.

Os resultados remotos observados para a temperatura superficial deste corpo de água nos meses de inverno mostram que os ventos fortes e a $\mathrm{TA}\left({ }^{\circ} \mathrm{C}\right)$ tendem a influenciar de forma a gerar maiores variações na TSL ao longo do perfil. As maiores flutuações da TSL estão sob a influência dos ventos mais fortes para os meses de inverno e para $\mathrm{TA}\left({ }^{\circ} \mathrm{C}\right)$ altas.

A passagem do $1^{\circ}$ sistema frontal, Fig. 9 mostra aumento da variação da TSL na entrada da frente, apresentando CV de 17,0 \%. A medida que o vento diminui, a TSL sofre queda e se estabiliza baixando seu CV para $3,41 \%$. No $3^{\circ}$ sistema frontal, Fig.11, observa-se a maior 
variação da TSL, CV de 23,19 \% na entrada da frente fria caracterizada por ventos da ordem de $4 \mathrm{~m} / \mathrm{s}$ e $\mathrm{TA}\left({ }^{\circ} \mathrm{C}\right)$ com média de $16,7^{\circ} \mathrm{C}$. Na passagem do $2^{\circ}$ e $4^{\circ}$ sistemas frontais, Figs. 10 e 12, também é observado na entrada e saída das frentes, menor variação da TSL associada com a baixa velocidade dos ventos da ordem de 1 e $2 \mathrm{~m} / \mathrm{s}$. Nestas situações, a energia do vento não gera movimentos turbulentos ondulatórios capazes de criar a mistura da coluna do corpo de água. Neste caso, não ocorrendo movimentos turbulentos a TSL se estabiliza. Assim, o efeito observado das altas variações da TSL associado com os ventos fortes e altas $\mathrm{TA}\left({ }^{\circ} \mathrm{C}\right)$ pode ser explicado pela energia do vento que gera movimentos turbulentos na superfície, que por sua vez produz os movimentos das correntes de fundo ocasionando a mistura das colunas de água. Desta forma, a TSL da superfície se torna mais instável no momento da captura da TSL pelo sensor MODIS.

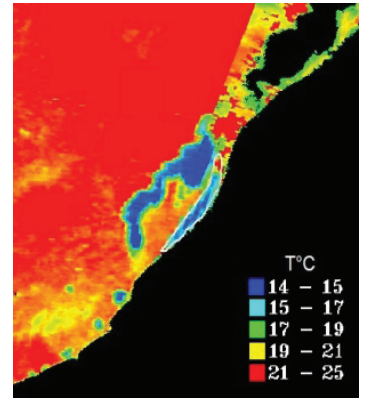

(a)

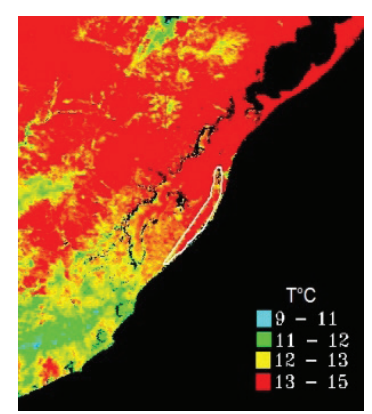

(b)
Figura 3 - 1ํ Sistema Frontal (a)08/06/06; (b) 12/06/06

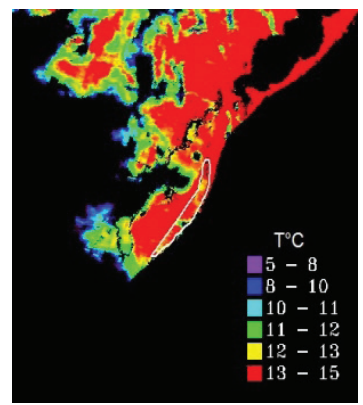

(a)

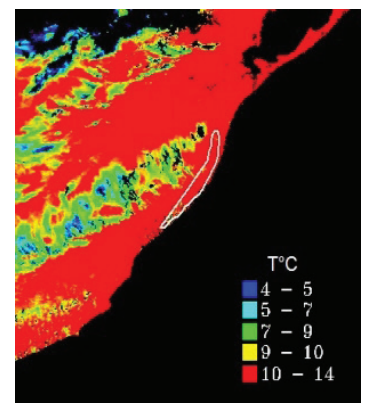

(b)
Figura 4 - 2o Sistema Frontal (a) 13/06/06; (b) 16/06/06

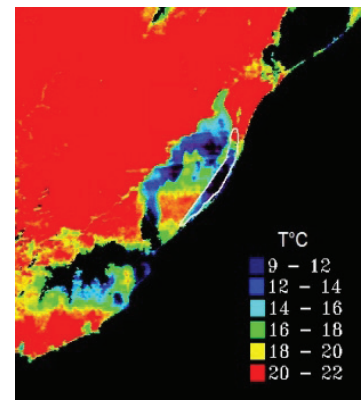

(a)

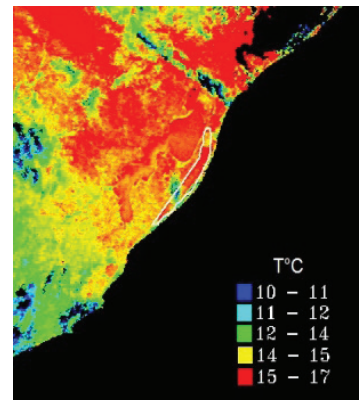

(b)

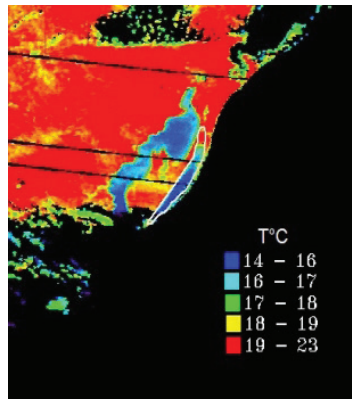

(a)

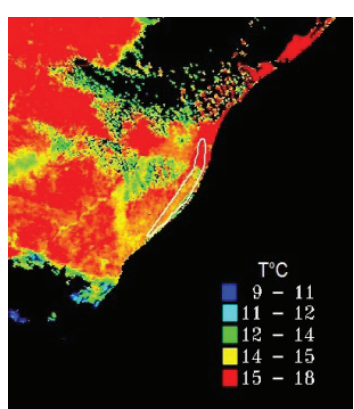

(b)
Figura 6 - 4º Sistema Frontal (a) 21/07/06; (b) 23/07/06

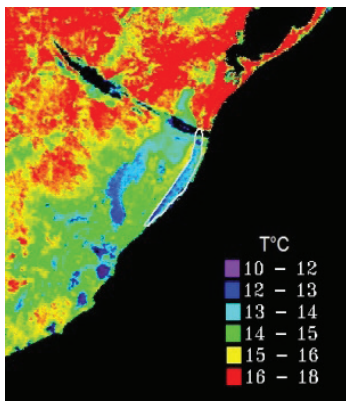

(a)

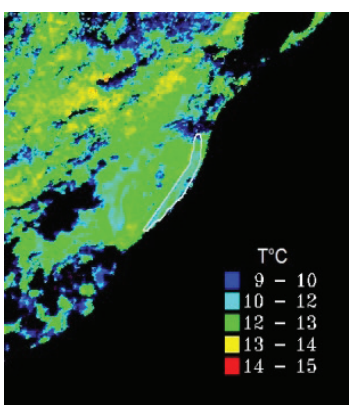

(b)
Figura 7 - 5을 Sistema Frontal (a) 19/08/06; (b) 21/08/06

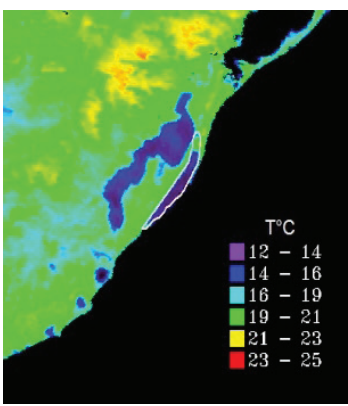

(a)

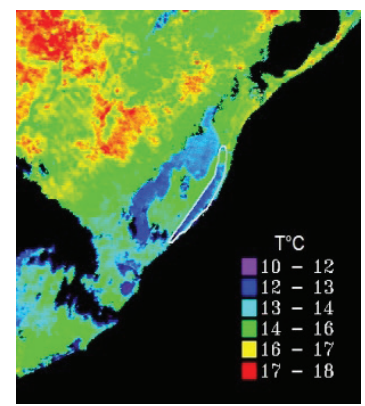

(b)
Figura 8 - 6o Sistema Frontal (a) 25/08/06; (b) 28/08/06

Tabela 4 - Estatísticas de temperatura de superfície do perfil Longitudinal PL da lagoa Mangueira na entrada e saída das frentes frias dos meses de inverno de 2006.

\begin{tabular}{|l|l|c|c|c|c|c|c|}
\hline \multicolumn{2}{|l|}{ Frente Fria } & Data & Máx & Mín & $\begin{array}{c}\bar{x} \\
\left({ }^{\circ} \mathrm{C}\right)\end{array}$ & $\begin{array}{c}\text { S } \\
\left({ }^{\circ} \mathrm{C}\right)\end{array}$ & $\begin{array}{c}\text { CV } \\
(\%)\end{array}$ \\
\hline \multirow{2}{*}{$1^{\text {a }}$} & Entrada & $08 / 06$ & 24,44 & 14,74 & 17,35 & 2,91 & 17,00 \\
\cline { 2 - 8 } & Saída & $12 / 06$ & 14,00 & 11,92 & 13,39 & 0,46 & 3,41 \\
\hline \multirow{2}{*}{$2^{\mathrm{a}}$} & Entrada & $13 / 06$ & 14,44 & 10,36 & 12,91 & 0,65 & 5,01 \\
\cline { 2 - 8 } & Saída & $16 / 06$ & 13,52 & 8,86 & 11,81 & 1,13 & 9,58 \\
\hline \multirow{2}{*}{$3^{\mathrm{a}}$} & Entrada & $07 / 07$ & 21,18 & 9,72 & 13,77 & 3,19 & 23,19 \\
\cline { 2 - 8 } & Saída & $09 / 07$ & 16,00 & 11,46 & 14,37 & 0,76 & 5,26 \\
\hline \multirow{2}{*}{$4^{\mathrm{a}}$} & Entrada & $21 / 07$ & 21,34 & 13,68 & 16,58 & 2,17 & 13,07 \\
\cline { 2 - 8 } & Saída & $23 / 07$ & 16,04 & 13,20 & 14,81 & 0,61 & 4,15 \\
\hline \multirow{2}{*}{$5^{\mathrm{a}}$} & Entrada & $19 / 08$ & 14,52 & 11,46 & 13,07 & 0,43 & 3,28 \\
\cline { 2 - 8 } & Saída & $21 / 08$ & 12,44 & 8,30 & 11,22 & 0,72 & 6,42 \\
\hline \multirow{2}{*}{$6^{\mathrm{a}}$} & Entrada & $25 / 08$ & 21,46 & 13,42 & 15,66 & 2,49 & 15,90 \\
\cline { 2 - 8 } & Saída & $28 / 08$ & 14,76 & 10,60 & 12,79 & 0,71 & 5,55 \\
\hline
\end{tabular}

Figura 5 - 3ํo Sistema Frontal (a) 07/06/06; (b) 09/06/06 
Tabela 5 - Estatísticas de temperatura de superfície da Máscara da lagoa Mangueira na entrada e saída das frentes frias dos meses de inverno de 2006

\begin{tabular}{|l|l|c|c|c|c|c|c|}
\hline \multicolumn{2}{|l|}{ Frente Fria } & Data & Máx & Mín & $\begin{array}{c}\bar{x} \\
\left({ }^{\circ} \mathrm{C}\right)\end{array}$ & $\begin{array}{c}\text { S } \\
\left({ }^{\circ} \mathrm{C}\right)\end{array}$ & $\begin{array}{c}\mathrm{CV} \\
(\%)\end{array}$ \\
\hline \multirow{2}{*}{$1^{\mathrm{a}}$} & Entrada & $08 / 06$ & 24,66 & 14,60 & 16,78 & 2,01 & 11,98 \\
\cline { 2 - 8 } & Saída & $12 / 06$ & 14,56 & 11,18 & 12,66 & 0,64 & 5,05 \\
\hline \multirow{2}{*}{$2^{\mathrm{a}}$} & Entrada & $13 / 06$ & 13,82 & 5,95 & 12,14 & 0,81 & 6,67 \\
\cline { 2 - 8 } & Saída & $16 / 06$ & 13,90 & 7,46 & 11,47 & 1,18 & 10,29 \\
\hline \multirow{2}{*}{$3^{\mathrm{a}}$} & Entrada & $07 / 07$ & 18,14 & 7,86 & 11,86 & 1,80 & 15,18 \\
\cline { 2 - 8 } & Saída & $09 / 07$ & 16,20 & 9,40 & 13,72 & 1,03 & 7,51 \\
\hline \multirow{2}{*}{$4^{\mathrm{a}}$} & Entrada & $21 / 07$ & 10,93 & 17,80 & 15,08 & 0,97 & 6,43 \\
\cline { 2 - 8 } & Saída & $23 / 07$ & 11,96 & 16,50 & 14,16 & 0,57 & 4,02 \\
\hline \multirow{2}{*}{$5^{\mathrm{a}}$} & Entrada & $19 / 08$ & 13,51 & 11,26 & 12,41 & 0,39 & 3,14 \\
\cline { 2 - 8 } & Saída & $21 / 08$ & 12,78 & 6,05 & 10,60 & 0,63 & 5,94 \\
\hline \multirow{2}{*}{$6^{\mathrm{a}}$} & Entrada & $25 / 08$ & 18,05 & 12,32 & 14,27 & 1,06 & 7,43 \\
\cline { 2 - 8 } & Saída & $28 / 08$ & 14,28 & 10,90 & 12,02 & 0,43 & 3,58 \\
\hline \multirow{2}{*}{} & & & & & &
\end{tabular}

$1^{\underline{a}}$ e $2^{\underline{a}}$ Frente fria (Junho/06)

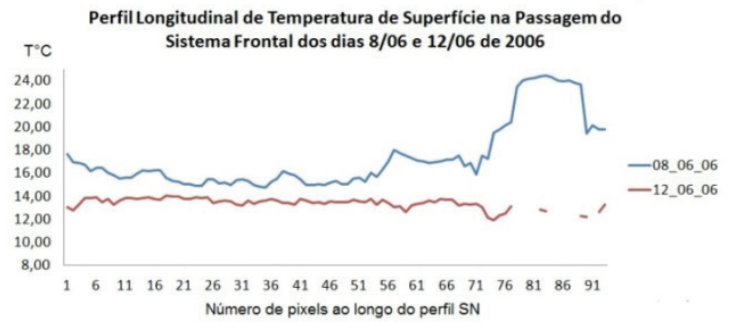

Figura 9 - Perfil L na passagem do $1^{\circ} \mathrm{SF}$

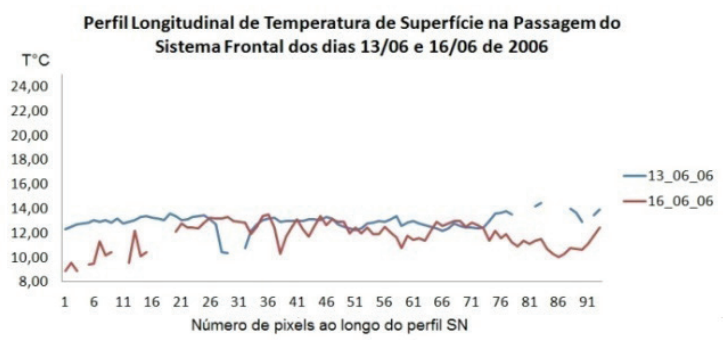

Figura 10 - Perfil L na passagem do $2^{\circ} \mathrm{SF}$

$3^{\text {a }}$ Frente fria e $4^{\underline{a}}$ Frente fria (julho/06)

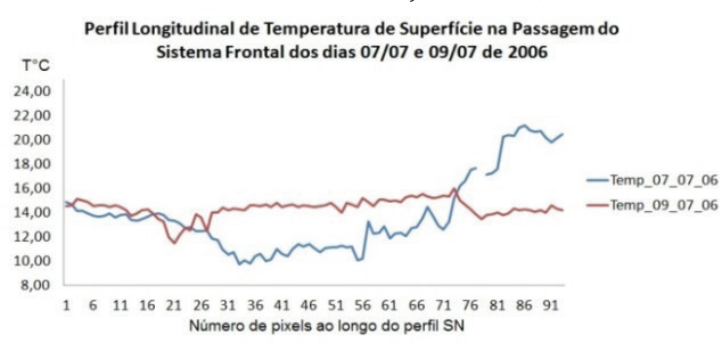

Figura 11 - Perfil L na passagem do $3^{\circ} \mathrm{SF}$

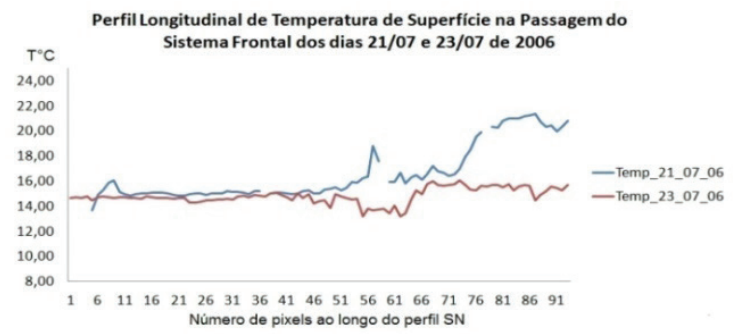

Figura 12 - Perfil L na passagem do $4^{\circ} \mathrm{SF}$

5 ${ }^{\underline{a}}$ Frente fria e $6^{\underline{a}}$ Frente fria (agosto/06)

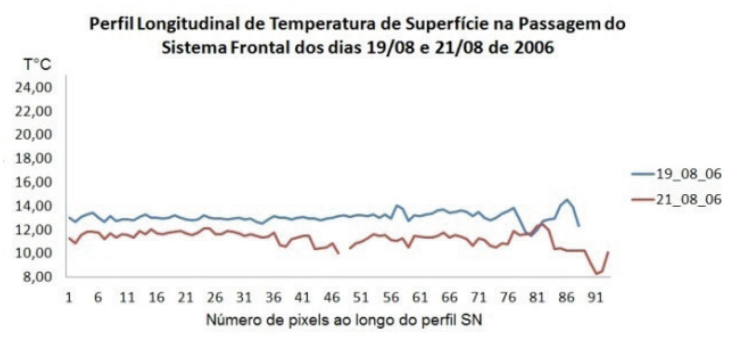

Figura 13 - Perfil L na passagem do $5^{\circ} \mathrm{SF}$

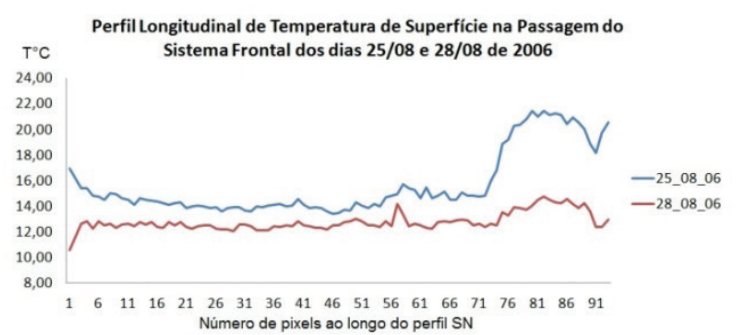

Figura 14 - Perfil L na passagem do $6^{\circ} \mathrm{SF}$

A investigação revela que a temperatura de superfície da lagoa Mangueira diminui após a passagem de frentes frias. Do ponto de vista quantitativo, considerando toda a superfície da lagoa, este decréscimo na temperatura é da ordem de $2^{\circ} \mathrm{C}$. Por outro lado, considerando o PL, a variação média é de $3^{\circ} \mathrm{C}$. Além disso, observou-se que existe uma relação entre os valores observados da temperatura superficial da lagoa com o vetor velocidade do vento (direção e magnitude) e com a temperatura ambiente. A presença de ventos fortes, com direções bem definidas, provoca grandes flutuações nas medidas da TSL. Estas advecções robustas, com magnitudes variando entre 5 e $8 \mathrm{~m} / \mathrm{s}$, são direcionalmente persistentes, ou seja, os ventos apresentam durante horas direções preferenciais consolidadas. Neste caso, as maiores flutuações de temperatura são consequência da turbulência da água, ocasionando assim maior instabilidade da TSL na superfície. A temperatura ambiente é outro fator que influencia a temperatura de superfície. Os resultados revelam que para TA $\left({ }^{\circ} \mathrm{C}\right)$ mais elevadas antes da passagem da frente 
fria ocorre maior variação da TSL. Este escoamento de ar quente turbulento provocado pelo vento, logo acima da lagoa, transfere energia para a superfície da água gerando turbulência e ondas. Como consequência, esta turbulência na superfície da lagoa pode ser o processo físico responsável por provocar as maiores flutuações observadas na TSL. Entretanto, quando a TA $\left({ }^{\circ} \mathrm{C}\right)$ apresenta valores baixos e a magnitude do vento é fraca, em curtos períodos de tempo, a sua direção apresenta uma grande variação. Tal fenômeno é conhecido como o meandro direcional horizontal do vento. Neste caso, as baixas velocidades do vento (advecção fraca), não geram turbulência atmosférica suficiente para gerar movimentos na superfície de água da lagoa. Este desacoplamento de energia, entre o contorno que separa os dois fluídos (ar e água), impede a geração de ondas e turbulência na superfície de água da lagoa. Neste caso, as flutuações de temperatura superficial observadas são reduzidas.

\section{Conclusões}

A determinação de temperaturas superficiais de lagoas costeiras, além de ser importante para avaliar os efeitos de mudanças climáticas, fornece as variações de temperatura que podem influenciar diferentes espécies biológicas presentes no ambiente da lagoa. A presente análise mostra que a temperatura superficial diminui após as passagens dos sistemas frontais e a metodologia utilizada permitiu identificar os escoamentos de água contendo diferentes materiais originados de ambientes aquáticos distintos da lagoa, como o caso do banhado do Taim. Além disso, o método de medidas remotas de temperatura superficial em lagoas, obtidas pelo sensor MODIS, constitui uma ferramenta viável e eficiente para estabelecer relações entre esta quantatidade e o campo de vento originado durante a passagem de sistemas frontais. A partir desta primeira verificação das mudanças da TSL, pode-se desenvolver trabalhos futuros com um maior aprofundamento relacionado à limnologia desta lagoa e à outros fatores meteorológicos que não foram considerados neste estudo.

\section{Bibliografia}

BECKER, F; LI, Z.L. Toward a local split-window method over land surfaces. International Journal of Remote Sensing, v. 11; n. 3; p. 369-393, 1990.

CLIMANÁLISE, Boletim de Monitoramento e Análise Climática, http://climanalise.cptec.inpe.br/ rclimanl/ boletim/index0706.shtml\#, acessado em maio de 2013.

CLIMANÁliSE, Boletim de Monitoramento e Análise Climática, Vol. $21-\mathrm{n}^{\circ}$ 06, Junho/2006. Cachoeira Paulista, SP, Brasil, INPE/CPTEC, 1986. Publicação Mensal.
ISSN 0103-0019 CDU-555.5 http://climanalise.cptec. inpe.br/ rclimanl/boletim/edicoes/2006/jun/fig22.shtml, acessado em maio de 2013.

CLIMANÁliSE, Boletim de Monitoramento e Análise Climática, Vol. $21-\mathrm{n}^{\circ}$ 07, julho/2006. Cachoeira Paulista, SP, Brasil, INPE/CPTEC, 1986. Publicação Mensal. ISSN 0103-0019 CDU-555.5 http://climanalise.cptec. inpe.br/ rclimanl/boletim/edicoes/2006/jul/fig22.shtml, acessado em maio de 2013.

CLIMANÁlise, Boletim de Monitoramento e Análise Climática, Vol. $21-\mathrm{n}^{\circ}$ 08, agosto/2005. Cachoeira Paulista, SP, Brasil, INPE/CPTEC, 1986. Publicação Mensal. ISSN 0103-0019 CDU-555.5. http://climanalise.cptec.inpe. br/ rclimanl/boletim/edicoes/2006/ago/fig22.shtmlacessado em maio de 2013.

DASH, P.; GOETTSCHE, F. M.; OLESEN, F. S.; FISHER, H. Land surface temperature and emissivity estimation from passive sensor data: theory and practice; current trends. International Journal of Remote Sensing, vol 23, n. 13, p. 2563-2594. 2002.

DELANEY PJV (1965) Fisiografia e geologia da superfície da planície costeira do Rio Grande do Sul. Publ Esp Esc Geol Univ Fed Rio Grande Sul (Porto Alegre) 6:1-63.

DOUSSET, B; GOURMELON, F. Satellite Multi-Sensor Data Analysis of urban surface temperatures and lancover. Isprs Journal of Photogrammetry e Romote Sensing, n. 58, p. 43-54, 2003.

FRAGOSO JR., C.R., MOTTA MARQUES, D.,COLLISCHONN, W. and VAN NES, E.H., 2008. Modelling spatial heterogeneity of phytoplankton in Lake Mangueira, a large shallow subtropical lake in South Brazil. Ecol. Model. Submmited.

GODOLPHIN MF (1976) Geologia do Holoceno costeiro do município de Rio Grande-RS. Tese de Mestrado, Uni Fed Rio Grande Sul, Brasil

KERR, Y.H., J.P.; LAGOUARD, J.; IMBERNON, I. Accurate land surface temperature retrieval from AVHRR data with use of an improved spit-window. Remote Sensing of Environment, v.. 41; p. 197-209. 1992

JUSTICE, C. O., TOWNSHEND, J. R. G., VERMOTE, E. F. MASUOKA, E., WOLFE, R. E., SALEOUS, N., ROY, D. P.,MORISSETE, J. T. An overview of modis land data processing and product status. Remote Sensing of Environment, n.83, p 3 - 15, 2002.

MOHSENI O. \& STEFAN H.G. (2001) Water budgets of two watersheds in different climatic zones under projected climate warming. Climate Change, 49, 77-104. 
MOHSENI O., STEFAN H.G. \& EATON J.G. (2003) Global warming and potential changes in fish habitat in U.S. streams. Climatic Change, 59, 389-409.

NASA. Site de aquisição das Imagens do produto Mod11. Disponível em http://reverb.echo.nasa.gov/reverb. Acessado em dezembro de 2011.

PRATA, J.A. Land Surface Temperature determination from satellites. Advances in space research, v. 14; n. 3; p. 15-26. March 1994.

RODRIGUES, L. H. R. (2009) PhD Thesis. Reguladores da dinâmica das comunidades planctônicas e íctica em ecossistemas límnicos subtropicais. Postgraduate program in water qualify management and environmental sanitation, instituto de pesquisas hidráulicas, universidade federal do rio grande do sul. Porto Alegre, Brazil.

SNYDER LH, GRIEVE KL, BROTCHIE P, ANDERSEN RA (1998) Separate bodyand world-referenced representations of visual space in parietal cortex. Nature 394:887-891.

SOBRINO, J.A.;LI, Z.-L.; STOLL, M.P.; BECKER, F. Multichannel and multi-angle algorithms for estimating sea and land surface temperature with ATSR data. International Journal of Remote Sensing, v. 17, p. 2089-2114. 1996

STEFAN H.G., FANG X. \& EATON J.G. (2001) Simulated fish habitat changes in North American lakes in response to projected climate warming. Transactions of the American Fisheries Society, 130, 459-477.

STRAHLER, A.; MUCHONEY, D.; BORAK, J.; FRIEDL, M.; GOPAL, S.; LAMBIN, E.; MOODY, A. (1999). MODIS Land Cover and Land-Cover Change products algorithm theoretical basis document (ATBD). Version 5.0, Center for Remote Sensing, Department of Geography, Boston University, May, 72p.

TOMMAZELLI LJ (1993) Regime dos ventos e a taxa de migração das dunas eólicas costeiras do Rio Grande do Sul, Brasil. Pesquisas (Porto Alegre) 20(1):18-26

TUNDISI, JG.; MATSUMURA-TUNDISI, T.; LUZIA, AP.; PASOERINI, MO.; CHIBA, W.; SEBASTIAN, NY. Cold fronts and reservoir limnology: an integrated approach towards the ecological dynamics of freshwater ecosystems. Brazilian Journal of Biology, v. 70, p. 815-824, 2010.

ULIVERI, C.; CASTRONUOVO, M.M.; FRANCIONI, R.; CARDILHO, A. A Split-window algorithm for estimating land surface temperature from satellites.
Advances in Space Research. v. 14; n. 3; p. 59-66. March 1994.

USTIN S L (2004) Remote Sensing for Natural Resource Management and Environment Monitoring. Third Edition, Volume 4, Pages 403-446.

ZELTZER F (1976) Geologia e paleogeografia da restinga da Laguna dos Patos, RS. Tese de Mestrado, Univ Fed Rio Grande Sul, Brasil

WAN, Z. AND J. DOZIER, “A generalized split-window algorithm for retrieving land-surface temperature from space," EEE Trans. Geosci. Remote Sens., vol. 34, no. 4, pp. 892-905, 1996. 